\section{GlaxoSmithKline}

HER2-positives metastasiertes Mammakarzinom

Lapatinib nach Trastuzumab-haltiger adjuvanter Chemotherapie

Der Tyrosinkinasehemmer Lapatinib ist eine potentiell wichtige neue Therapieoption für Patientinnen mit Trastuzumab-resistentem HER2-positivem, metastasiertem Mammakarzinom. Diese erläuterte José Baselga anlässlich des San Antonio Breast Cancer Symposiums (SABCS) 2005. Er sieht die Möglichkeit, Lapatinib bei diesen Patientinnen mit Trastuzumab zu kombinieren. Da Lapatinib die Aktivität der Tyrosinkinase sowohl über den ErbB1- als auch den ErbB2-Rezeptor hemmt, greift die Substanz auf zwei molekularen Wegen in die Pathogenese der Tumorproliferation ein. Neben der eigenständigen antineoplastischen Wirkung unterstützt Lapatinib auch die Wirkung von Trastuzumab. In-vitro-Untersuchungen zeigen synergistische Effekte.

In klinischen Studien erreichte Lapatinib als Monosubstanz bei über $30 \%$ der Patientinnen mit HER2-positivem metastasiertem Mammakarzinom eine objektive Tumorrückbildung. Auch Trastuzumabresistente Patientinnen sprachen auf Lapatinib an. Für die Kombination Lapatinib/Trastuzumab spricht, dass unter Invitro-Bedingungen synergistische Effekte zwischen beiden Substanzen nachgewiesen wurden, die sich klinisch bestätigten. In einer klinischen Phase-I-Studie einer Studiengruppe um Sporniolo et al. wurden insgesamt 54 meist intensiv vorbehandelte Patientinnen mit HER2-positivem metastasiertem Mammakarzinom und Rezidiv nach Trastuzumab-haltiger Vortherapie mit Lapatinib/Trastuzumab behandelt. Die eine Hälfte der Patientinnen $(\mathrm{n}=27)$ wurde im Rahmen einer Dosiseskalationsstudie behandelt. Bei fixer Trastuzumab-Standard-Dosierung wurde die Dosis von Lapatinib von initial $1.000 \mathrm{mg} / \mathrm{Tag}$ auf maximal $1.500 \mathrm{mg} / \mathrm{Tag}$ erhöht bzw. ggf. auf $750 \mathrm{mg} / \mathrm{Tag}$ reduziert. Die andere Hälfte der Patientinnen wurde bei einer Lapatinib-Dosierung von $1.000 \mathrm{mg} /$ Tag einer pharmakokinetischen Untersuchung unterzogen, um eventuelle Interaktionen zwischen Lapatinib und Trastuzumab zu untersuchen.

Trotz der ungünstigen Rahmenbedingungen - intensive Vorbehandlung, weit fortgeschrittenen Erkrankung - profitierten 19 der 54 Patientinnen (35\%) von der Behandlung mit Lapatinib/Trastuzumab. Im Rahmen der Dosiseskalationsstudie erzielten $26 \%$ der Patientinnen (7/27) eine objektive Tumorrückbildung, darunter auch eine Patientin mit kompletter Remission, die 14 Monate andauerte. 3 weitere Patientinnen hatten einen Wachstumsstop des Tumors (Stabilisierung der Erkrankung), der über ein halbes Jahr anhielt, so dass bei insgesamt 37\% (10/27) der Patientinnen die weitere Progression der Erkrankung deutlich hinausgezögert wurde. Als optimal tolerables
Regime (OTR) erwiesen sich 1.000 mg/Tag Lapatinib plus die wöchentliche Trastuzumab-Gabe. Unter dieser Dosierung traten keine inakzeptablen Nebenwirkungen auf. Im Vordergrund standen gastrointestinale Beschwerden, Müdigkeit und Hautrötungen. Bei keiner Patientin kam es zu einem symptomatischen Abfall der linksventrikulären Ejektionsfraktion (LVEF). Im Rahmen der pharmakokinetischen Studie erzielten 9 der 27 Patientinnen (33\%) mindestens eine Krankheitsstabilisierung von über 6 Monaten. Die Kombination beider Substanzen führte zu keiner gegenseitigen pharmakokinetischen Beeinflussung.

Bei Patientinnen mit raschem Rezidiv nach adjuvanter Trastuzumab-haltiger Chemotherapie besteht in der Regel ein unverzüglicher Therapiebedarf, betonte Baselga, insbesondere dann, wenn eine symptomatische Metastasierung vorliegt. Die Kombination Lapatinib/Trastuzumab ist aufgrund der synergistischen Wirkung beider Substanzen eine viel versprechende Option für diese Frauen. Ist die rezidivfreie Zeitspanne nach Trastuzumab-haltiger adjuvanter Chemotherapie länger als 6 Monate, empfiehlt Baselga für die First-line-Therapie Trastuzumab plus eine konventionelle Chemotherapie; Lapatinib ist dann eine Option für die Second-line-Therapie.

Weitere Informationen bei

GlaxoSmithKline GmbH \& Co. KG

Sonja Luz, Wissenschafts-PR

Theresienhöhe 11

80339 München

Tel.: +49-(0)89-36044-8256, Fax -98256

sonja.luz@gsk.com

www.glaxosmithkline.de

\title{
PharmaTicker+++ PharmaTicker+++ PharmaTicker+++ PharmaTicker+++
}

Novartis Pharma GmbH. Der Aromatasehemmer Letrozol (Femara ${ }^{\circledR}$ ) hat in den USA die Zulassung für die adjuvante Therapie des hormonsensitiven Mammakarzinoms im Frühstadium nach einer Operation bei postmenopausalen Frauen erhalten.

Weitere Informationen be

Novartis Pharma GmbH

Dr. Michaela Paudler-Debus, Leiterin Kommunikation

Tel. +49 911 273-12462, Fax -12971

michaela.paudler-debus@novartis.com
Schering Deutschland GmbH. Die neue Patientenbroschüre «Mehr Lebensqualität für Krebspatienten mit Knochenmetastasen: Schmerzlinderung mit der Radionuklidtherapie» informiert über Einsatz und Nutzen von Quadramet ${ }^{\circledR}$. Die Broschüre ist zu beziehen bei

Schering Deutschland $\mathrm{GmbH}$

Max-Dohrn-Str. 10

10589 Berlin
MSD MERCK SHARP \& DOHME GMBH.

Wie jetzt in einer Studie gezeigt wurde, kann Aprepitant (EMEND ${ }^{\circledR}$ ) in Kombination mit anderen Antiemetika sowohl bei hoch emetogenen cisplatinhaltigen als auch bei moderat emetognen Chemotherapien über alle Zyklen hinweg vor Übelkeit und Erbrechen schützen. Weitere Informationen bei

MSD MERCK SHARP \& DOHME GMBH

Annette Menzel

Lindenplatz 1

85540 Haar

Tel. +49 89 4561-1353, Fax -1329

anette_menzel@msd.de

\begin{tabular}{ll}
\hline KARGER & @ 2006 S. Karger GmbH, Freiburg \\
& \\
$\begin{array}{l}\text { Fax + 49 761 452 } 5714 \\
\begin{array}{l}\text { E-mail Information@Karger.de } \\
\text { www.karger.com }\end{array}\end{array}$ & $\begin{array}{l}\text { Acessible online at: } \\
\text { www.karger.com/brc }\end{array}$ \\
&
\end{tabular}




\section{ब仿 \\ sanofi aventis}

Das Wichtigste ist die Gesundheit

\section{BCIRG 006: Taxotere $^{\circledR}+$ Herceptin $^{\circledR}$ verringern Rezidivrisiko bei Brust- krebs im Frühstadium}

Die Breast Cancer International Research Group (BCIRG) und Sanofi-Aventis haben die Ergebnisse der ersten Zwischenanalyse zur Wirksamkeit und der aktualisierten Sicherheitsanalyse der Phase-III-Brustkrebsstudie BCIRG 006 bekannt gegeben.

In die Studie wurden zwischen März 2001 und Februar 2004 insgesamt 3.222 Frauen mit HER2-postivem Brustkrebs im Frühstadium aufgenommen. Die Frauen, die für die Aufnahme in die Studie geeignet waren, wurden nach primärer Operation und Lymphknotendissektion nach dem Zufallsprinzip einem der drei folgenden Behandlungsarme zugeteilt:

- einer Standardbehandlung aus $4 \mathrm{Zy}$ klen Doxorubicin und Zyklophosphamid gefolgt von Taxotere für 4 Zyklen (AC-T),

- einem experimentellen Arm des oben genannten Regimes in Kombination mit 1 Jahr Behandlung mit Herceptin, gleichzeitig beginnend mit Taxotere (AC-TH) und

- einem weiterens Arm mit 6 Zyklen Taxotere und Carboplatin mit $1 \mathrm{Jahr}$ Behandlung mit Herceptin (TCH). Im letzteren Arm wurde die HerceptinBehandlung gleichzeitig mit der Chemotherapie begonnen.
Die Daten zur kardialen und allgemeinen Sicherheit zusammen mit der Zwischenanalyse zur Wirksamkeit auf der Grundlage von 322 Fällen wurden von einem «Independent Data Monitoring Committee» überprüft. Die relative Verringerung des Rezidivrisikos betrug: $51 \%$ [95\% CI: $35-63 \%$ ], bei einem p-Wert von $<0,001$ und 39\% [95\% CI: 21-53\%], bei einem pWert von < 0,001 für den AC-TH- bzw. TCH-Arm der Studie im Vergleich zum Kontrollarm AC-T. Es gab keine statistisch signifikante Differenz zwischen den beiden Herceptin ${ }^{\circledR}$-enthaltenen experimentellen Armen. Die Daten zum Gesamtüberleben sind noch nicht verfügbar.

Sanofi-Aventis Deutschland GmbH

Cristina Iannazzo

Industriepark Höchst, Gebäude F821

65926 Frankfurt am Main

cristina.iannazzo@sanofi-aventis.com

\section{AstraZeneca ONKOLOGIE}

\section{Überlebensvorteil in der Adjuvanz mit Arimidex ${ }^{\circledR}$}

Anastrozol schreibt erneut Geschichte: Es ist der erste Aromatasehemmer, der einen signifikanten Überlebensvorteil in der Adjuvanz zeigt: Werden mit Tamoxifen behandelte Brustkrebspatientinnen auf Anastrozol umgestellt, haben sie eine um 29\% höhere Überlebenschance als bei Fortführung der Tamoxifen-Therapie.
Auf dem 28. San Antonio Breast Cancer Symposium wurden die Ergebnisse einer Meta-Analyse, für die man die Daten aller 4.006 Patientinnen der Studien ITA, ARNO 95 und ABCSG 8 gemeinsam ausgewertet hat, vorgestellt. Die drei Studien sind alle bereits publiziert. Sie zeigen jede für sich eine signifikante Verlängerung des krankheitsfreien Überlebens und ein geringeres Risiko für das Auftreten von Fernmetastasen, wenn postmenopausale Frauen mit hormonsensitivem Brustkrebs nach 2-3 Jahren adjuvanter Tamoxifen-Therapie auf Anastrozol umgestellt werden. Die neue Metaanalyse belegt nun auch:
Patientinnen, die auf Anastrozol umgestellt wurden, haben im Vergleich zu den kontinuierlich mit Tamoxifen behandelten Frauen ein signifikant um 29\% verringertes Sterberisiko. Wie in den Einzelstudien führte die Umstellung auf den Aromatasehemmer außerdem $\mathrm{zu}$ einer Verbesserung des ereignisfreien Überlebens und des fernmetastasenfreien Überlebens. Bislang unbekannte Nebenwirkungen wurden nicht beobachtet.

Weitere Informationen bei

AstraZeneca $\mathrm{GmbH}$

Peter Schiffer

Tinsdaler Weg 183, 22880 Wedel

Tel. +49 4103 708-3663, Fax -3663

peter.schiffer@astrazeneca.com

\section{PharmaTicker+++ PharmaTicker+++ PharmaTicker+++ PharmaTicker+++}

Pierre Fabre Pharma GmbH. Navelbine ${ }^{\circledR}$ Oral (Vinorelbin oral) ist seit November 2005 zur Behandlung des fortgeschrittenen anthrazyklinresistenten Mammakarzinoms für Patientinnen in gutem Allgemeinzustand in Deutschland zugelassen und als Weichkapsel in den Wirkstärken 20 und $30 \mathrm{mg}$ erhältlich.

Weitere Informationen bei

Pierre Fabre Pharma GmbH, Onkologie

Tel. +49761452610
Hoffmann-La Roche. Ein neuer Film «Wenn Brustkrebs wiederkommt» wendet sich an Frauen mit rezidiviertem oder metastasiertem Brustkrebs. Er ergänzt die Kampagne «Durch die Brust ins Herz", die von der Firma Hoffmann-La Roche unterstützt wird und kann kostenlos bezogen werden unter www.brustkrebszentrale.de oder bei Durch die Brust ins Herz Postfach 511170 50947 Köln ribosepharm GmbH. «Das Menschenmögliche tun» - mit diesem Slogan präsentiert sich ribosepharm auf seiner erweiterten Internetseite www.ribosepharm.de, wo Patienten ausführliches Informationsmaterial zum Thema Krebs finden und sich medizinische Fachkreise detailliert über die ribosepharm-Produkte informieren können.

Weitere Informationen be

POMME-med GmbH, Dr. Helga Pilz

Tel. + 4989 454566-22, Fax -27

pilz@pomme-med.de

\begin{tabular}{ll}
\hline KARGER & @ 2006 S. Karger GmbH, Freiburg \\
& \\
$\begin{array}{l}\text { Fax +49 761 452 } 5714 \\
\begin{array}{l}\text { E-mail Information@Karger.de } \\
\text { www.karger.com }\end{array}\end{array}$ & $\begin{array}{l}\text { Accessible online at: } \\
\text { www.karger.com/brc }\end{array}$ \\
&
\end{tabular}

